\title{
The CDKL5 disorder is an independent clinical entity associated with early-onset encephalopathy
}

\author{
Stephanie Fehr ${ }^{1}$, Meredith Wilson ${ }^{2,3}$, Jenny Downs ${ }^{1,4}$, Simon Williams ${ }^{5}$, Alessandra Murgia ${ }^{6}$, Stefano Sartori ${ }^{6}$, \\ Marilena Vecchi ${ }^{6}$, Gladys $\mathrm{Ho}^{2,3}$, Roberta Polli ${ }^{6}$, Stavroula Psoni ${ }^{7}$, Xinhua Bao ${ }^{8}$, Nick de Klerk ${ }^{1}$, Helen Leonard ${ }^{1}$ \\ and John Christodoulou ${ }^{\star 2,3}$
}

The clinical understanding of the CDKL5 disorder remains limited, with most information being derived from small patient groups seen at individual centres. This study uses a large international data collection to describe the clinical profile of the CDKL5 disorder and compare with Rett syndrome (RTT). Information on individuals with cyclin-dependent kinase-like 5 (CDKL5) mutations $(n=86)$ and females with MECP2 mutations $(n=920)$ was sourced from the InterRett database. Available photographs of CDKL5 patients were examined for dysmorphic features. The proportion of CDKL5 patients meeting the recent Neul criteria for atypical RTT was determined. Logistic regression and time-to-event analyses were used to compare the occurrence of Rett-like features in those with MECP2 and CDKL5 mutations. Most individuals with CDKL5 mutations had severe developmental delay from birth, seizure onset before the age of 3 months and similar non-dysmorphic features. Less than one-quarter met the criteria for early-onset seizure variant RTT. Seizures and sleep disturbances were more common than in those with MECP2 mutations whereas features of regression and spinal curvature were less common. The CDKL5 disorder presents with a distinct clinical profile and a subtle facial, limb and hand phenotype that may assist in differentiation from other early-onset encephalopathies. Although mutations in the CDKL5 gene have been described in association with the early-onset variant of RTT, in our study the majority did not meet these criteria. Therefore, the CDKL5 disorder should be considered separate to RTT, rather than another variant.

European Journal of Human Genetics (2013) 21, 266-273; doi:10.1038/ejhg.2012.156; published online 8 August 2012

Keywords: CDKL5; Rett syndrome; dysmorphology; natural history; phenotype

\section{INTRODUCTION}

Mutations in the cyclin-dependent kinase-like 5 (CDKL5) gene have been found in individuals diagnosed with the early-onset seizure variant of Rett syndrome (ESV RTT), infantile spasms or West syndrome. In 2003, chromosomal rearrangements involving the CDKL5 gene were identified in two females with severe intellectual disability and early-onset seizures ${ }^{1}$ and the following year in four females with Rett syndrome (RTT). ${ }^{2,3}$ To date, 141 individuals (127 females and 14 males) with a CDKL5 mutation have been described, mostly within small case series, ${ }^{1-38}$ the largest including 20 patients. ${ }^{11}$ Therefore, the understanding of the CDKL5 disorder profile is still limited and a larger series is required to further understand its presentation and natural history.

The clinical characteristics commonly associated with a CDKL5 mutation include early-onset seizures and severe intellectual and gross motor impairment. The ESV RTT was originally described in a girl with RTT who developed seizures by 1 year of age. ${ }^{39}$ The recently published Neul criteria ${ }^{40}$ for atypical RTT included five specific items (early-onset seizures before 5 months of age, infantile spasms, refractory myoclonic epilepsy, seizure onset before regression and decreased frequency of typical RTT features) for differentiating the ESV from other atypical forms. The number of distinguishing criteria required for a diagnosis of ESV RTT was not clear, although testing for a CDKL5 mutation was recommended.

Various dysmorphic features have also been described in individuals with a CDKL5 mutation including large deep-set eyes, strabismus, high forehead, full lips, wide mouth and widely spaced teeth. $1,4,5,7,13,18,20,22,25,26,28-30,35$ The presence of typical facial or other features could provide additional assistance in the clinical identification of individuals with a CDKL5 mutation.

Here we describe the phenotype of females and males with a pathogenic or potentially pathogenic CDKL5 mutation, identified through the International Rett Syndrome Phenotype Database (InterRett). ${ }^{41}$ We assessed whether individuals with a CDKL5 mutation shared similar characteristics of the face, hands and feet. We determined whether these individuals met the latest criteria for ESV RTT ${ }^{40}$ and compared the presence of RTT features in females who have a CDKL5 mutation with females who have a pathogenic MECP2 mutation.

${ }^{1}$ Telethon Institute for Child Health Research, Centre for Child Health Research, The University of Western Australia, Perth, Western Australia, Australia; ${ }^{2}$ Western Sydney Genetics Program, Children's Hospital at Westmead, Sydney, New South Wales, Australia; ${ }^{3}$ Disciplines of Paediatrics \& Child Health and Genetic Medicine, University of Sydney, Sydney, New South Wales, Australia; ${ }^{4}$ School of Physiotherapy and Curtin Health Innovation Research Institute, Curtin University, Perth, Western Australia, Australia; ${ }^{5}$ Department of Neurology and Rehabilitation, Princess Margaret Hospital, Perth, Western Australia, Australia; ${ }^{6}$ Department of Pediatrics, University of Padua, Padua, Italy; ${ }^{7}$ Medical Genetics, University of Athens, Athens, Greece; ${ }^{8}$ Department of Pediatrics, Peking University First Hospital, Beijing, PRC

*Correspondence: Professor J Christodoulou, Western Sydney Genetics Program and Sydney Medical School, University of Sydney, Sydney, NSW, Australia; Tel: +612 9845 3452; Fax: +612 9845 1864; E-mail: john.christodoulou@health.nsw.gov.au

Received 17 January 2012; revised 14 May 2012; accepted 8 June 2012; published online 8 August 2012 


\section{METHODS}

InterRett is an international RTT database that has ascertained over 2000 RTT cases worldwide since $2003 .{ }^{41}$ Since 2007 , partly through the advocacy of the family support group, families of an individual with a CDKL5 mutation have been invited to complete an InterRett questionnaire. Families also provided photographs of their son or daughter's face (frontal and side), hands (dorsal and palmar) and feet (dorsal and plantar). A comparison group of females with RTT and a pathogenic MECP2 mutation was also obtained from the InterRett database.

Data from family and clinician questionnaires, concerning acquisition of gross motor milestones, purposeful hand function and speech, presence of hand stereotypies, seizures, gastrointestinal problems, spinal curvature (scoliosis or kyphosis), autonomic problems and sleep problems were examined. All photographs of patients with CDKL5 mutations were reviewed by a clinical geneticist (MW) and the characteristics noted were tabulated.

Approval for this study was provided by the Princess Margaret Hospital for Children Ethics Committee, Perth, Western Australia, Australia.

\section{Statistical analysis}

The phenotypical characteristics of the CDKL5 disorder and the difference between females and males were determined using descriptive statistics. The proportion of CDKL5 disorder individuals that met each of the criteria for ESV RTT, by sex and age (aged 5 years and under or over 5 years), was determined. Clinical features known to be associated with RTT were compared between females with a CDKL5 mutation and females with a MECP2 mutation. Odds ratios adjusting for age were estimated. Survival analysis was used to investigate the timing of onset of seizures, stereotypies and spinal curvature.

\section{RESULTS}

Seventy-seven females and nine males with a CDKL5 mutation were included in this study (Supplementary Table 1 contains genetic information). Age at ascertainment ranged from 6 months to 22.4 years (mean 6.1 years, median 4.7 years) for females and from 1.1 to 14.9 years (mean 5.2 years, median 4.6 years) for males.

\section{Seizures and motor delay}

Seizures occurred in all except one female, and in all males, at a mean age of 7.3 weeks (range $0.3-34.8$ ) and 6.4 weeks (2.1-13.0 weeks), respectively. Seizures occurred by 3 months of age in $90 \%$. At ascertainment, 52/72 females and 8/9 males had daily seizures, 5/72 females had weekly seizures, 10/72 females and 1/9 males had monthly seizures, while the remaining five females had no seizures in the last year. Nine families reported that their child was no longer treated with anticonvulsant medications.

Severe gross motor delay was reported in all but three females. The ability to sit was acquired by just over half $(41 / 75,54.7 \%)$ of the females (mean age 25.2 months, median 18.2 months, range 6 months to 14 years) and by one male at 8 months. Eight (10.8\%) females achieved independent walking at a mean age of 39.6 months (95\% CI 28.2-51.1, and median 38 months, range 1.4-5.5 years). A further nine (13.6\%) females walked with assistance (mean age 41.2 months, 95\% CI 26.4-55.9, range 1.6-5 years). No males were able to walk, with or without assistance.

\section{Hand function and speech}

Functional hand use was acquired by just over half (43/72) of females and $1 / 8$ males, of whom $11 / 37(29.8 \%)$ females and no males lost the ability. Early speech skills (ranging from babble to single words) were acquired by $30 / 76(39.5 \%)$ females and $2 / 9(22.2 \%)$ males, of whom $14 / 29$ (48.3\%) females and no males subsequently lost speech. Families of 57/72 females and 6/8 males felt that their child could communicate non-verbally (eg, facial expressions, sign language and/or eye pointing) and families of 56/72 females and 6/8 males felt their child was able to follow simple commands and respond to changes in tone of voice.

\section{RTT-like features and co-morbidities}

Hand stereotypies developed in 61/76 (80.3\%) females (mean age 13.1 months, median 1 year, range 3 months to 5.5 years). In comparison only $3 / 9(33.3 \%)$ males developed stereotypies at 3, 12 and 24 months $(P=0.004)$. In the majority $(41 / 56,73.2 \%)$ more than one type of hand stereotypy was present, the most frequently described being mouthing $(n=36 / 56)$, wringing $(n=18 / 56)$, clapping $(n=18 / 56)$, biting $(n=12 / 56)$ and clasping $(n=11 / 56)$. Regression in skills occurred in $25 / 77$ (32.5\%) females (mean age of 18.5 months) and no males.

Gastrointestinal problems, such as constipation and reflux, were present in $59 / 75(78.7 \%)$ of females and in 8 males. A spinal curvature was reported in $20.5 \%(15 / 73)$ of females (mean age of onset (where known) 7.6 years, range 2-14 years) and one-third of the males (age range 4-13 years). Autonomic problems, such as cold feet, were present in just under half of females $(30 / 73,41.1 \%)$ and $3 / 8$ of males, while sleep problems occurred in $89.9 \%(62 / 69)$ of females and $87.5 \%$ (7/8) males. Head growth deceleration occurred in 25/42, however, only 3 of these would be classified as having microcephaly.

\section{Dysmorphism}

Initial review of photographs (60 females and 7 males) indicated that 'dysmorphism' was subtle. Frequently observed facial features included: a prominent and/or broad forehead; high hairline; relative mid-face hypoplasia; deep-set but 'large'-appearing eyes and infraorbital shadowing (Figure 1). Eyebrows were usually well defined and tended to be straight rather than arched. Synophrys was present in $13.4 \%$ overall but was more prevalent in males (42.9\%). Lips were full in most, often with eversion of the lower lip. Ears were normally placed and of normal configuration. Young children tended to have a low nasal bridge, mildly anteverted nares, prominent nasal tip and a well-defined (normal) philtrum (Table 1). With age the facial appearance tended to coarsen, but the nasal profile remained notably straight in nearly all individuals.

The fingers in young children were often tapered, some with puffy proximal and narrowed distal phalanges. Some had a puffy dorsum of the hands and/or feet. In older individuals, the fingers tended to be slender, with prominent proximal interphalangeal (IP) joints and narrow distal IP joints. Hallux valgus was present in $25 \%$. Some had relatively long great toes, with slightly broad toenails. Toes $2-5$ were usually very regular in shape and position, with mild brachydactyly (Table 1).

The spectrum of features was similar overall in females and males, but 5/7 males had a distinctly anteverted nasal tip, two of whom had a short philtrum and retracted, everted upper lip. Only one female had anteverted nares; she also had a receding forehead and laterally upswept eyebrows; she had a $2.7 \mathrm{Mb}$ deletion including the CDKL5 and NHS genes. The mutation type and features present in nontypical individuals is shown in Table 2.

\section{Fulfilment of criteria for ESV RTT}

Overall 19/75 (23.7\%) females and no males fulfilled all criteria for ESV RTT (Table 3). This was largely due to the absence of regression in all males and $67.5 \%$ of females. Hand function was lost by only $16.7 \%$ and spoken language by only $18.7 \%$ of females. No male lost either hand function or spoken language. 


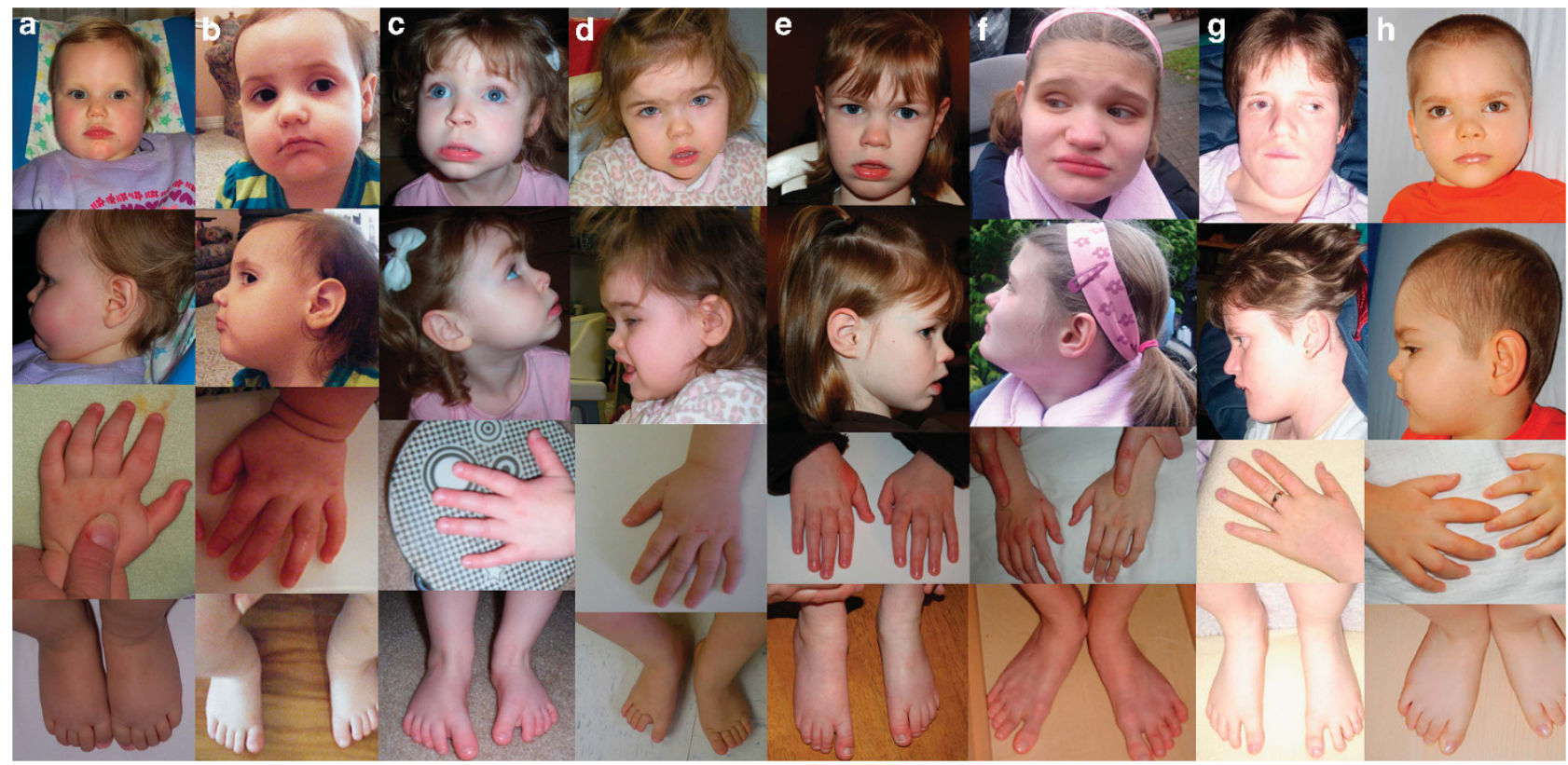

Figure 1 Examples of identified facial, hand and feet features in males and females with the CDKL5 disorder ( $n=67)$. (a) Female aged 1 year and 10 months: high/prominent forehead, deep-set eyes, prominent lips, well-defined philtrum, proximal puffy phalanges and tapered fingers. (b) Female aged 2 years: high/prominent forehead, deep-set eyes, prominent lips, well-defined philtrum, tapered fingers, slightly broad hallux, hallux valgus and 'regular' toes. (c) Female aged 4 years: deep-set eyes, a well-defined philtrum, laterally orientated nasal apertures, prominent lips, slightly tapered fingers, small distal phalanges and a broad hallux. (d) Female aged 3 years and 2 months: prominent forehead, mild synophrys, deep-set eyes, prominent lips, puffy proximal phalanges, tapered fingers, slightly broad hallux and 'regular' other toes. (e) Female aged 7 years and 11 months: high/prominent forehead, deep-set eyes, everted lower lip, puffy proximal phalanges, prominent proximal IP joints and tapered fingers. (f) Female aged 14 years: broad forehead, deep-set eyes, epicanthic folds, well-defined philtrum, prominent lips, tapered fingers and prominent proximal IP joints. (g) Female aged 24 years and 2 months: high forehead, deep-set eyes, well-defined philtrum and prominent lips. (h) Male aged 4 years 4 months: high/prominent forehead, well-defined philtrum, prominent lips, tapered fingers and 'regular' toes.

Table 1 Distribution of observed dysmorphic features identified in 60 females and 7 males with the CDKL5 disorder

\begin{tabular}{lc} 
Feature & Number of cases (\%) \\
\hline Broad/prominent forehead & $46 / 62(74.2)$ \\
High forehead (not broad) & $5 / 62(8.1)$ \\
Receding or narrow forehead & $3 / 62(4.8)$ \\
Deep-set eyes & $49 / 67(73.1)$ \\
Synophrys (five females, three males) & $8 / 67(13.4)$ \\
Well-defined philtrum & $40 / 66(60.6)$ \\
Full lips/everted lower & $45 / 67(67.2)$ \\
Fingers proximally puffy/prominent proximal IP joint & $26 / 62(41.9)$ \\
Hallux valgus & $16 / 64(25.0)$
\end{tabular}

Abbreviations: CDKL5, cyclin-dependent kinase-like 5; IP, interphalangeal.

Gait abnormalities were the most widely met main criterion. Bruxism, impaired sleep and abnormal muscle tone were the most widely met supportive criteria.

More females aged over 5 years (12/36) met all criteria in comparison with those aged 5 years and under (7/39) (Table 3). Just under half of the females aged over 5 years had developed a spinal curvature, compared with $<5 \%$ of those aged 5 years and under. In males all those aged over 5 years had a spinal curvature compared with none of those $<5$ years. Peripheral vasomotor disturbances were also reported more frequently in the older group in both females and males.
Table 2 Mutation type in females and males with non-typical dysmorphic features

\begin{tabular}{ll}
\hline Mutation & Features \\
\hline $\begin{array}{l}\text { Females } \\
\text { c.2376 + 5G > A (p.K760fs, }\end{array}$ & Receding forehead \\
exon 16 skipping) & \\
c.2154_2155insGT (p.P719fs) & High narrow forehead \\
arr Xp22.2p22.13(16 256335- & Receding forehead and anteverted \\
18963 757) (loss of CDKL5 and & pointy nose \\
NHS gene) & \\
Males & \\
c.473G $>$ C (p.R158P) & \\
c.175C $>$ T (p.R59X) & Retracted upper lip, short philtrum and \\
& $\begin{array}{l}\text { anteverted, hypoplastic nasal tip } \\
\text { Retracted upper lip, short philtrum and } \\
\text { anteverted pointy nose }\end{array}$ \\
\hline
\end{tabular}

Comparison between females with the CDKL5 disorder and females with RTT

There were a total of 920 females with a clinical diagnosis of RTT and a pathogenic MECP2 mutation. Data were available from both families and clinicians $(n=574)$ and from clinicians only (in de-identified form, $n=346$ ). The mean age of the females with RTT was 10.5 years (range 1.3-54.2 years). After adjusting for age, females with the CDKL5 disorder were more likely to have seizures (OR 54.01, 
Table 3 The proportion of females $(n=77)$ and males $(n=9)$ that met each of the latest criteria for ESV RTT 40

\begin{tabular}{|c|c|c|c|c|c|c|}
\hline \multirow[b]{3}{*}{2010 ESV RTT criteria } & \multicolumn{6}{|c|}{ Number of CDKL5-positive cases } \\
\hline & \multicolumn{3}{|c|}{ Female } & \multicolumn{3}{|c|}{ Male } \\
\hline & $\leq 5$ Years & $>5$ Years & All & $\leq 5$ Years & $>5$ Years & $A / l$ \\
\hline \multicolumn{7}{|l|}{ Necessary criteria } \\
\hline A period of regression followed by recovery or stabilisation & $10 / 41(24.4)$ & $15 / 36(41.7)$ & $25 / 77(32.5)$ & $1 / 6(16.7)$ & $0 / 3(0.0)$ & $1 / 9(11.1)$ \\
\hline \multicolumn{7}{|l|}{ Main criteria } \\
\hline Partial or complete loss of acquired purposeful hand function & 4/36 (11.1) & $7 / 30(23.3)$ & $11 / 66(16.7)$ & $0 / 5(0.0)$ & $0 / 3(0.0)$ & $0 / 8(0.0)$ \\
\hline Partial or complete loss of acquired spoken language & $8 / 40(20.0)$ & $6 / 35(17.1)$ & $14 / 75(18.7)$ & $0 / 6(0.0)$ & $0 / 3(0.0)$ & $0 / 9(0.0)$ \\
\hline Gait abnormalities: Impaired (dyspraxic) or absence of ability & $40 / 40(100.0)$ & $27 / 34(79.4)$ & $67 / 74(90.5)$ & $6 / 6(100.0)$ & $3 / 3(100.0)$ & 9/9 (100.0) \\
\hline Stereotypic hand movements & $32 / 41(78.0)$ & $29 / 35(82.9)$ & $61 / 76(80.3)$ & $3 / 6(50.0)$ & $0 / 3(0.0)$ & $3 / 9(33.3)$ \\
\hline \multicolumn{7}{|l|}{ Supportive criteria } \\
\hline Breathing disturbances when awake & $19 / 38(50.0)$ & $17 / 33(51.5)$ & $36 / 71(50.7)$ & $3 / 6(50.0)$ & $2 / 3(66.7)$ & $5 / 9(55.5)$ \\
\hline Bruxism when awake & $33 / 40(82.5)$ & $30 / 33(90.9)$ & $63 / 73(86.3)$ & $5 / 5(100.0)$ & $3 / 3(100.0)$ & $8 / 8(100.0)$ \\
\hline Impaired sleep pattern & $31 / 36(86.1)$ & $31 / 33(93.9)$ & $62 / 69(89.8)$ & $5 / 5(100.0)$ & $2 / 3(66.7)$ & $7 / 8(87.5)$ \\
\hline Abnormal muscle tone & $13 / 15(86.7)$ & $10 / 11(90.0)$ & $23 / 26(88.5)$ & $2 / 2(100.00)$ & NA & $2 / 2(100.0)$ \\
\hline Peripheral vasomotor disturbances & $11 / 40(27.5)$ & $19 / 33(57.6)$ & $30 / 73(41.1)$ & $1 / 5(20.0)$ & $2 / 3(66.7)$ & $3 / 8(37.5)$ \\
\hline Scoliosis/kyphosis & $1 / 39(2.6)$ & $14 / 34(41.2)$ & $15 / 73(20.5)$ & $0 / 5(0.0)$ & $3 / 3(100.0)$ & $3 / 8(37.5)$ \\
\hline Growth retardation & $6 / 10(60.0)$ & $6 / 9(66.7)$ & $12 / 19(63.1)$ & $0 / 2(0.0)$ & Missing & $0 / 2(0.0)$ \\
\hline Small cold hands or feet & $19 / 39(48.7)$ & $24 / 31(77.4)$ & $43 / 70(61.4)$ & $2 / 5(40.0)$ & 2/3 (66.7) & $4 / 8(50.0)$ \\
\hline Inappropriate laughing/screaming spells & $22 / 35(62.9)$ & $29 / 32(90.6)$ & $51 / 67(76.1)$ & $3 / 5(50.0)$ & $1 / 3(33.3)$ & $4 / 8(50.0)$ \\
\hline Diminished response to pain & $15 / 37(40.5)$ & $11 / 31(35.5)$ & $26 / 68(38.2)$ & $0 / 5(0.0)$ & $1 / 3(33.3)$ & $1 / 8(12.5)$ \\
\hline Intense eye communication 'eye pointing' & $5 / 37(13.5)$ & $5 / 33(15.1)$ & $10 / 70(14.3)$ & $0 / 5(0.0)$ & $0 / 3(0.0)$ & $0 / 8(0.0)$ \\
\hline \multicolumn{7}{|l|}{ ESV-specific criteria } \\
\hline Early-onset seizures before 5 months of age & $40 / 40(100.0)$ & $32 / 35(91.4)$ & $72 / 75(96.0)$ & $6 / 6(100.0)$ & $3 / 3(100.0)$ & 9/9 (100.0) \\
\hline Infantile spasms ${ }^{a}$ & $11 / 22(50.0)$ & $3 / 14(21.4)$ & $14 / 36(38.9)$ & $1 / 3(33.3)$ & $1 / 1(100.0)$ & $2 / 4(50.0)$ \\
\hline Refractory myoclonic epilepsy ${ }^{b}$ & & & $N A$ & & & \\
\hline Seizure onset before regression & $40 / 41(97.6)$ & $30 / 36(83.3)$ & 70/77 (90.9) & $4 / 5(80.0)$ & $3 / 3(100.0)$ & $7 / 8(87.5)$ \\
\hline Decreased frequency of typical RTT features ${ }^{c}$ & & & NA & & & \\
\hline \multicolumn{7}{|l|}{ Meets criteria for atypical RTT } \\
\hline Necessary criteria & $10 / 41(24.4)$ & $15 / 36(41.7)$ & $25 / 77(32.5)$ & $1 / 6(16.7)$ & $0 / 3(0.0)$ & $1 / 9(11.1)$ \\
\hline Two of the four main criteria & $32 / 40(80.0)$ & $25 / 33(75.8)$ & $57 / 73(79.5)$ & $3 / 6(50.0)$ & $0 / 3(0.0)$ & $3 / 9(33.3)$ \\
\hline 5 Of the 11 supportive criteria & $20 / 28(71.4)$ & $26 / 26(100.0)$ & $46 / 54(86.4)$ & $2 / 3(66.7)$ & $3 / 3(100.0)$ & $5 / 6(83.3)$ \\
\hline All criteria & $7 / 39(17.9)$ & $12 / 36(33.3)$ & $19 / 75(23.7)$ & $0 / 6(0.0)$ & $0 / 3(0.0)$ & $0 / 9(0.0)$ \\
\hline
\end{tabular}

Abbreviations: CDKL5, cyclin-dependent kinase-like 5; Cl, confidence interval; ESV RTT, early-onset seizure variant of Rett syndrome; NA, not applicable. aThe occurrence of infantile spasms was determined from the clinician questionnaire or if the family had listed infantile spasms as their child's diagnosis.

bOur questionnaire did not contain enough information to be able to determine this criterion.

'Decreased frequency of typical RTT features was not able to be determined as it is unclear which features are included and what is considered a 'decreased' frequency.

95\% CI 7.45-391.33) and sleep disturbances (OR 4.89, 95\% CI 2.20-10.89) than females with RTT (Table 4). They were less likely to have breathing disturbances (OR $0.4395 \%$, CI $0.26-0.71$ ), a spinal curvature (OR 0.34, 95\% CI 0.17-0.65) and gastrointestinal problems (OR 0.52, 95\% CI 0.27-0.98); to develop hand stereotypies (OR 0.12, 95\% CI 0.06-0.25) and to lose hand (OR 0.06, 95\% CI 0.03-0.12) and speech skills (OR 0.13, 95\% CI 0.07-0.23).

Time-to-event analysis (Figures 2-4) showed that females with the CDKL5 disorder were more likely to develop seizures earlier, with three-quarters of females having developed epilepsy before 12 months of age compared with 10 years for RTT (HR 24.8, 95\% CI 18.6-32.9). Hand stereotypies also appeared earlier in females with the CDKL5 disorder, with $75 \%$ having developed them by 2 years of age compared with $75 \%$ by 3 years for females with RTT (HR 1.1, 95\% CI 0.86-1.5). A spinal curvature was less likely in females with the CDKL5 disorder compared with females with RTT (HR 0.51, 95\% CI 0.28-0.90).
The most characteristic features of females and males with the CDKL5 disorder are shown in Table 5. These features can be used as a guide to assist decision-making where targeted CDKL5 mutation analysis is being considered.

\section{DISCUSSION}

Individuals with mutations in the CDKL5 gene have been variably classified as having early infantile epileptic encephalopathy, X-linked dominant infantile spasm syndrome, the ESV RTT or diagnosed with other epileptic disorders such as West syndrome. ${ }^{1,3}$ We described the clinical presentation and physical appearance of individuals with the CDKL5 disorder, providing information from a large international dataset. The CDKL5 disorder appears to be characterised by seizure onset in the majority before 3 months of age, severely impaired gross motor, language and hand function skills, and subtle but shared physical characteristics such as a prominent/broad forehead, deep-set but large-appearing eyes, full lips and tapered fingers. Our findings 
Table 4 Comparison of the occurrence of Rett syndrome features in 77 females with the CDKL5 disorder and 920 females with a pathogenic MECP2 mutation

\begin{tabular}{|c|c|c|c|c|}
\hline Rett syndrome characteristics & $\begin{array}{l}\text { Number of MECP2-positive } \\
\text { cases (\%) }\end{array}$ & $\begin{array}{l}\text { Number of CDKL5-positive } \\
\text { cases (\%) }\end{array}$ & Odds ratio $(95 \% \mathrm{Cl})$ & Age adjusted odds ratio (95\% Cl) \\
\hline Breathing disturbances & $602 / 829(72.6)$ & $36 / 71(50.7)$ & $0.39(0.24-0.63)$ & $0.43(0.26-0.71)$ \\
\hline Gastrointestinal problems & $511 / 564(90.6)$ & $59 / 75(78.7)$ & $0.38(0.20-0.71)$ & $0.52(0.27-0.98)$ \\
\hline Spinal curvature identified & $432 / 830(52.0)$ & $15 / 73(20.5)$ & $0.24(0.13-0.43)$ & $0.34(0.17-0.65)$ \\
\hline Sleep disturbances & $513 / 766(67.0)$ & $62 / 69$ (89.9) & $4.37(1.97-9.68)$ & $4.89(2.20-10.89)$ \\
\hline Abnormal muscle tone & 208/254 (81.9) & 23/27 (85.2) & $1.27(0.42-3.80)$ & $1.29(0.42-4.00)$ \\
\hline Autonomic disturbances & $459 / 783(58.6)$ & $30 / 73(41.1)$ & $0.49(0.30-0.80)$ & $0.67(0.40-1.10)$ \\
\hline Head deceleration & 252/398 (63.3) & 25/42 (59.5) & $0.85(0.44-1.63)$ & $0.92(0.47-1.77)$ \\
\hline Hand stereotypies & $815 / 838(97.3)$ & $61 / 76(80.3)$ & $0.11(0.06-0.23)$ & $0.12(0.06-0.25)$ \\
\hline Bruxism & 666/781 (85.3) & $63 / 73(86.3)$ & $1.09(0.54-2.18)$ & $1.08(0.53-2.18)$ \\
\hline Seizures & $545 / 853(63.9)$ & 76/77 (98.7) & $42.95(5.94-310.38)$ & $49.95(7.58-398.11)$ \\
\hline Loss of hand function & $627 / 795$ (78.9) & $11 / 66(16.7)$ & $0.05(0.03-0.10)$ & $0.06(0.03-0.12)$ \\
\hline Loss of spoken language & $568 / 844(67.3)$ & $14 / 75(18.7)$ & $0.11(0.06-0.20)$ & $0.13(0.07-0.23)$ \\
\hline Gait abnormalities & $736 / 845$ (87.1) & 67/74 (90.5) & $1.42(0.63-3.17)$ & $1.52(0.68-3.42)$ \\
\hline
\end{tabular}

Abbreviations: CDKL5, cyclin-dependent kinase-like 5; Cl, confidence interval.

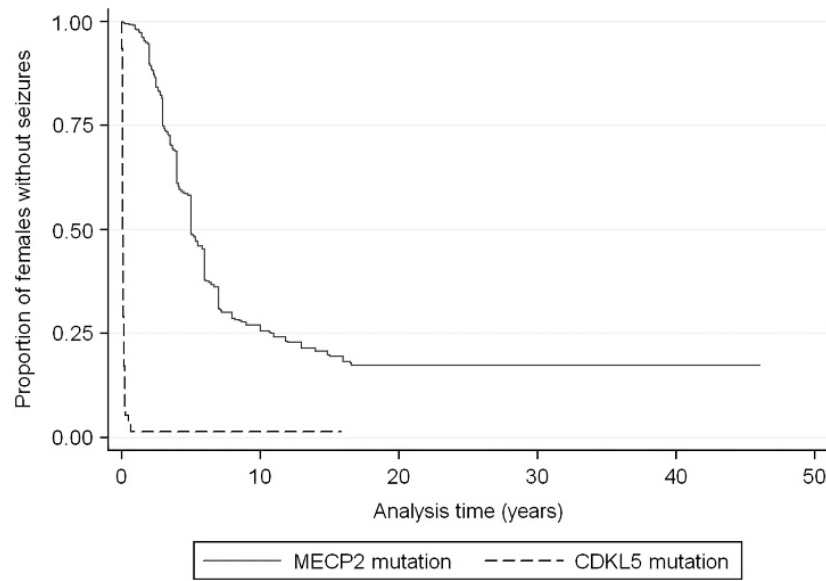

Figure 2 Kaplan-Meier survival curve for the risk of developing seizures by single year of age.

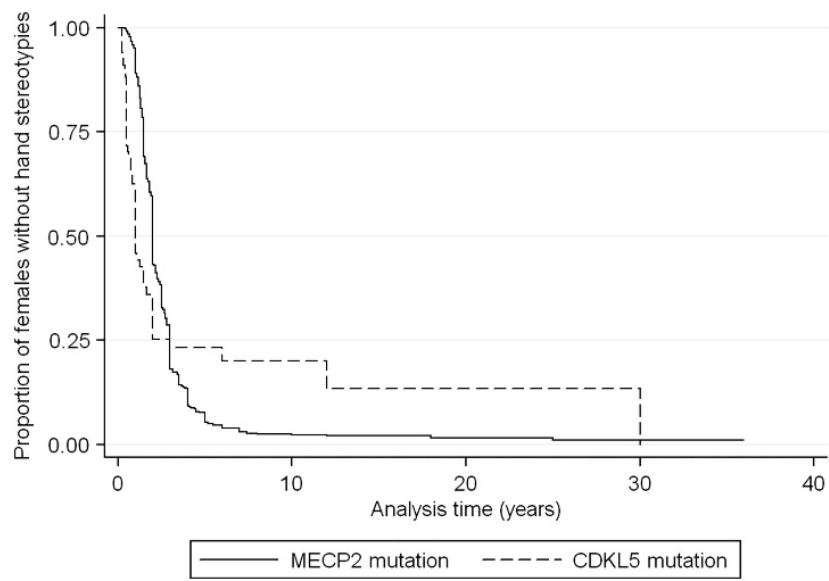

Figure 3 Kaplan-Meier survival curve of the risk of developing hand stereotypies by single year of age.

suggest that the CDKL5 disorder is an independent entity and should not be considered part of the RTT spectrum, as $<25 \%$ of the cases in our study would meet the clinical criteria for ESV RTT. Therefore, we

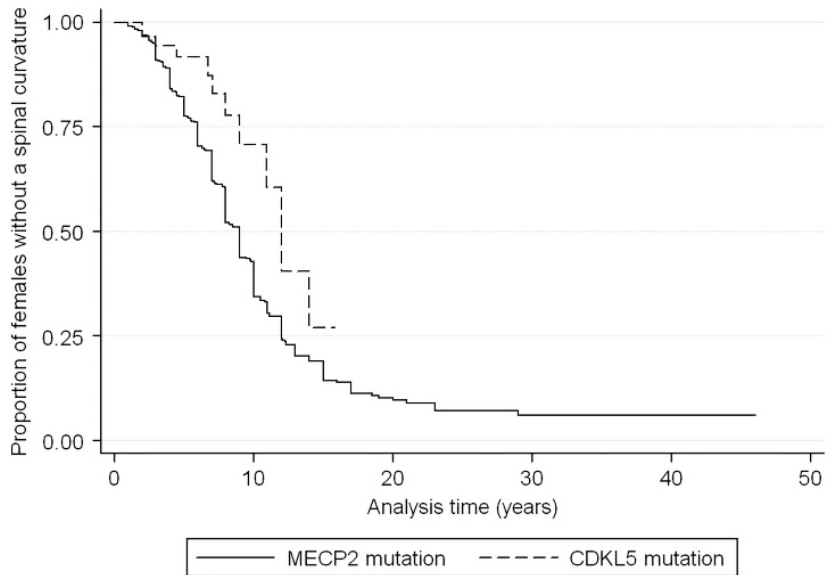

Figure 4 Kaplan-Meier survival curve for the risk of developing a spinal curvature by single year of age.

propose that females and males with a mutation in the CDKL5 gene be given a specific diagnosis of the CDKL5 disorder.

Our study found that early-onset epilepsy was one of the hallmark features of the CDKL5 disorder. In the literature many seizure types and EEG changes have been described. These include infantile spasms, multifocal and generalised seizures with myoclonic, tonic (tonic vibratory) and clonic features. Reported EEG patterns include a normal background, background slowing and a burst-suppression pattern. ${ }^{10,12}$ Although no specific seizure semiology has been described, two reports have noted a seizure pattern that may be unique to this disorder. ${ }^{31,34}$ The first $(n=4)$ reported a seizure pattern of tonic-tonic/vibratory contraction, followed by a clonic phase with spasms and finishing with distal myoclonus. ${ }^{34}$ The second $(n=4)$ reported an initial hypermotor phase, then tonic extension, followed by spasms. Severe developmental delay was typical of the CDKL5 disorder. Learning to sit and walk was usually considerably delayed or not achieved, more so in males. ${ }^{42}$ Communication was usually restricted to non-verbal strategies. It would be important to further investigate the influences of genotype, seizure severity or therapy utilisation on early development in those with the CDKL5 disorder. Consistent with other literature ${ }^{1-38}$ our large case series 
Table 5 Clinical features suggesting a diagnosis of the CDKL5 disorder

\begin{tabular}{ll}
\hline Extremely likelya $^{\text {a }}$ & Seizures within the first year of life (90\% by 3 months) \\
& Global developmental delay \\
& Severely impaired gross motor function \\
Very likely & Sleep disturbances \\
& Abnormal muscle tone \\
& Bruxism \\
& Gastrointestinal issues \\
& Subtle dysmorphic features including three or more of the \\
following: broad/prominent forehead; large 'deep-set' eyes; & full lips; tapered fingers; and anteverted nares in males \\
& Hand stereotypies \\
& Laughing and screaming spells \\
& Cold hands or feet \\
& Breathing disturbances \\
& Peripheral vasomotor disturbances \\
&
\end{tabular}

abserved in $>90 \%$ of cases in the current study.

bobserved in $80-90 \%$ of cases in the current study.

cObserved more variably in $40-80 \%$ of cases in the current study.

dObserved in $<10 \%$ of cases in the current study.

confirms that early-onset of epilepsy and severe developmental delay are key features.

Males with the CDKL5 disorder in this study tended to be more severely affected. None learned to walk, with or without assistance, and few acquired spoken communication and hand function. Seizure onset was slightly earlier than in females, and all males were having either daily or monthly seizures. Hand stereotypies were reported in only a third of males compared with $80 \%$ of females. Some of these differences may be due to amelioration of the phenotype in females because of X-inactivation resulting in a mosaic expression of normal and mutant CDKL5 protein. In RTT, males tend to be either extremely affected, often dying at an early age due to severe infantile encephalopathy, or more mildly affected than females. ${ }^{43}$ Those who are more mildly affected tend to have hypomorphic MECP2 mutations that are not found in females with RTT. It would be of interest to further investigate the genetic variability between males and females with the CDKL5 disorder to determine whether a similar phenomenon exists.

We observed a shared physical resemblance between females with the CDKL5 disorder, with the most consistent features being a prominent/broad forehead, deep-set but 'large-appearing' eyes, full lips and tapered fingers. Most affected males were more obviously dysmorphic, with distinctly anteverted nares, and several males also had a short philtrum and everted upper lip. Previous studies of the CDKL5 disorder have also reported dysmorphic features including: large deep-set eyes, strabismus, high forehead, full lips, wide mouth, widely spaced teeth and a high palate. ${ }^{1,4,5,7,13,18,20,22,25,26,28-30,35}$ Dysmorphic features have been described in other conditions presenting with early-onset encephalopathy, such as those with FOXG1 mutations and in Pitt-Hopkins syndrome (PHS). In those with FOXG1 mutations, subtle, non-specific dysmorphic features have been reported, along with severe microcephaly, which is typical of FOXG1 syndrome but not common in individuals with the CDKL5 disorder. ${ }^{44}$ The facial features in PHS include a high nasal bridge and prominent, beaked nose, different from the consistently straight nose and normal or low nasal bridge seen in the CDKL5 disorder. Although both can have prominent lips, the lips in PHS have a more marked cupid's-bow contour. Individuals with PHS may have clubbing of the fingertips whereas in the CDKL5 disorder the distal phalanges are relatively narrow and/or short. ${ }^{45}$ In classical RTT no typical facial gestalt has been described, although some clinicians have suggested a facial similarity to Angelman syndrome. ${ }^{46}$ A recent study using a combination of measurement and subjective impression found the only distinctive facial profile difference in RTT was a relatively broad upper face, especially in girls $<3$ years old. ${ }^{47}$ Although subtle, the features that we have described appear to be characteristic of the CDKL5 disorder population and could therefore be useful in determining whether the patient warranted CDKL5-specific mutation testing.

The majority of patients in this study with the CDKL5 disorder did not meet the new criteria for the ESV RTT, mainly because of the absence of regression in over two-thirds. Several of the specific supportive criteria, such as diminished response to pain, a spinal curvature and intense eye pointing, were also infrequently reported. Some of these supportive characteristics appear age-dependent and were observed more commonly in those aged over 5 years. Longitudinal monitoring of these features would contribute to a better understanding of the evolution of this disorder.

Considering only the females with the CDKL5 disorder, the differences in comparison to RTT persisted. Early development was more severely impaired in those with the CDKL5 disorder, with approximately half learning to sit and $10 \%$ learning to walk in comparison with $\sim 80 \%$ learning to sit and just under half learning to walk in a recent study of 293 females with RTT. ${ }^{48}$ Those with the CDKL5 disorder were less likely to use words whereas use of some words was acquired by nearly $90 \%$ of females with RTT before regression. ${ }^{48}$ Females with the CDKL5 disorder were also less likely to develop hand stereotypies, breathing disturbances, gastrointestinal problems and a spinal curvature, but were much more likely to develop seizures and have sleep disturbances. These findings again suggest that the CDKL5 disorder is clinically separate to RTT, indicating the need to develop independent clinical criteria for the identification of the CDKL5 disorder.

The two largest studies conducted to date $(n=9$ and $n=20)$ found that the classical period of regression was rarely present in females with the CDKL5 disorder and that autonomic, gastrointestinal, breathing and spinal problems were rare and lack of eye contact, hypotonia and epilepsy common. ${ }^{11,22}$ Our study also found that regression, autonomic disturbances, breathing disturbances and a spinal curvature occurred less frequently than in RTT, but that the prevalence increased with age. Gastrointestinal problems were quite common, although occurring less frequently than in females with RTT. In contrast to other CDKL5 studies, ${ }^{1-38}$ we found that sleep problems were reported frequently. Small case series may be less likely to accurately identify some of the patterns detectable by a larger study, which could account for this difference. Clearly large sample sizes, preferably population-based, are needed to provide accurate representation of the clinical features of such a rare condition. Our study is the first to have had the capacity to compare the characteristics of males and females with the CDKL5 disorder. Further investigation of the natural history is still required, and there are likely other features that have not been captured by existing studies. 
This study is the first international case collection investigating the clinical presentation of the CDKL5 disorder and includes more than four times the number of individuals than the largest previously reported case series. It is also the first to examine whether these individuals meet the criteria set forth for the ESV RTT. However, our study is not population-based, and we suggest the development of a population-based register of this disorder, as has occurred in RTT. ${ }^{49}$ Because we used the infrastructure of InterRett, our data collection tools were initially designed for RTT and therefore we may not have been able to capture the presence of features that are unique to the CDKL5 disorder. Our study population was also weighted towards younger children, and some clinical features are likely to evolve with age. With regard to the identification of 'dysmorphic' features, our study was limited by the lack of a photographic control group, the variable quality of images used and the subjective nature of the assessments (although the latter has been a traditional approach to dysmorphology). Acknowledging these limitations, and that the features noted were variable, we felt the combined gestalt in a child with early-onset encephalopathy, without major malformations, could suggest a CDKL5 mutation as a likely underlying aetiology.

Many of the early-onset encephalopathies have overlapping phenotypes, including severe developmental compromise with or without prior regression, early-onset seizures, abnormal movements (stereotypies or dyskinesias) and respiratory irregularities. Some are distinctive either by dysmorphism (PHS), or typical neurodevelopmental profile (classical RTT). Others may be less easily recognised, which is why classifications evolve using terms such as 'Rett variant' or 'PHS-like'. Although use of such terms in some conditions (eg, Noonan or Noonan-like) has been borne out by discovering mutations in genes in shared pathways, this type of classification should be approached with some caution. Although mutations in the CDKL5 gene have been found in association with a clinical picture similar to RTT in some instances, the majority of cases are different. It may be more accurate and beneficial for families and clinicians if the CDKL5 disorder is considered independent of RTT, rather than another variant. Therefore, we suggest that when describing the clinical picture of females and males with the CDKL5 disorder, researchers should not only concentrate on features that should be present in RTT, but also on the features that are present in the CDKL5 disorder. Only then will it be possible to develop accurate clinical diagnostic and management guidelines.

\section{CONFLICT OF INTEREST}

The authors declare no conflict of interest.

\section{ACKNOWLEDGEMENTS}

We thank all the families and clinicians who have contributed to the International Rett Syndrome Phenotype Database (InterRett) by completing questionnaires. We acknowledge the International Rett Syndrome Foundation (IRSF previously IRSA) for their ongoing support of the InterRett project and the International Foundation for CDKL5 Research for their support of this study and all the families, especially Leita Boltwood, who have actively worked in promoting this study. The InterRett project receives funding from the International Rett Syndrome Foundation (IRSF previously IRSA). Dr Helen Leonard is funded from an NHMRC Senior Research Fellowship no. 572568 and Stephanie Fehr receives funding from the University of Western Australia as part of her Australian Postgraduate Award and Safety-Net Top-Up Scholarship. This research was also part funded by NHMRC Project Grant no. 570752 and Dr Murgia, Dr Sartori and Dr Polli received funding from Fondazione Cariplo (Grant 2010-0724).
1 Kalscheuer VM, Tao J, Donnelly A et al: Disruption of the serine/threonine kinase 9 gene causes severe X-linked infantile spasms and mental retardation. Am J Hum Genet 2003; 72: 1401-1411.

2 Tao J, Van Esch H, Hagedorn-Greiwe $\mathrm{M}$ et al: Mutations in the X-linked cyclin dependent kinase-like 5 (CDKL5/STK9) gene are associated with severe neurodevelopmental retardation. Am J Hum Genet 2004; 75: 1149-1154.

3 Weaving LS, Christodoulou J, Williamson SL et al: Mutations of CDKL5 cause a severe neurodevelopmental disorder with infantile spasms and mental retardation. Am J Hum Genet 2004; 75: 1079-1093.

4 Evans JC, Archer HL, Colley JP et al: Early onset seizures and Rett-like features associated with mutations in CDKL5. Eur J Hum Genet 2005; 13: 1113-1120.

5 Archer $\mathrm{HL}$, Evans J, Edwards S et al: CDKL5 mutations cause infantile spasms, early onset seizures, and severe mental retardation in female patients. J Med Genet 2006, 43: 729-734.

6 Bertani I, Rusconi L, Bolognese $\mathrm{F}$ et al: Functional consequences of mutations in $C D K L 5$, an X-linked gene involved in infantile spasms and mental retardation. J Biol Chem 2006; 281: 32048-32056.

7 Jansen A, Bauters M, De Rademaeker M et al: Epileptic encephalopathy in a boy with an interstitial deletion of Xp22 comprising the CDKL5 gene. Epilepsia 2006; 47 367-367.

8 Nectoux J, Heron D, Tallot M, Chelly J, Bienvenu T: Maternal origin of a novel C-terminal truncation mutation in CDKL5 causing a severe atypical form of Rett syndrome. Clin Genet 2006; 70: 29-33.

9 Van Esch H, Jansen A, Bauters M, Froyen G, Fryns JP: Encephalopathy and bilateral cataract in a boy with an interstitial deletion of Xp22 comprising the CDKL5 and NHS genes. Am J Med Genet 2007; 143A: 364-369.

10 Grosso S, Brogna A, Bazzotti S, Renieri A, Morgese G, Balestri P: Seizures and electroencephalographic findings in CDKL5 mutations: case report and review. Brain Dev 2007; 29: 239-242.

11 Bahi-Buisson N, Nectoux J, Rosas-Vargas $\mathrm{H}$ et al: Key clinical features to identify girls with CDKL5 mutations. Brain 2008; 131: 2647-2661.

12 Bahi-Buisson N, Kaminska A, Boddaert N et al: The three stages of epilepsy in patients with CDKL5 mutations. Epilepsia 2008; 49: 1027-1037.

13 Elia M, Falco M, Ferri R et al: CDKL5 mutations in boys with severe encephalopathy and early-onset intractable epilepsy. Neurology 2008; 71: 997-999.

14 Rosas-Vargas H, Bahi-Buisson N, Philippe C et al: Impairment of CDKL5 nuclear localisation as a cause for severe infantile encephalopathy. J Med Genet 2008; 45 . 172-178.

15 Fichou $\mathrm{Y}$, Bieth $\mathrm{E}$, Bahi-Buisson $\mathrm{N}$ et al: Re: CDKL5 mutations in boys with severe encephalopathy and early-onset intractable epilepsy. Neurology 2009; 73: 77-78, (author reply 78)

16 Nemos C, Lambert L, Giuliano F et al: Mutational spectrum of CDKL5 in early-onset encephalopathies: a study of a large collection of French patients and review of the literature. Clin Genet 2009; 76: 357-371.

17 Psoni S, Willems PJ, Kanavakis E et al: A novel p.Arg970X mutation in the last exon of the CDKL5 gene resulting in late-onset seizure disorder. Eur J Paediatr Neurol 2010; 14: 188-191.

18 Russo S, Marchi M, Cogliati $\mathrm{F}$ et al: Novel mutations in the CDKL5 gene, predicted effects and associated phenotypes. Neurogenetics 2009; 10: 241-250.

19 Saitsu H, Osaka H, Nishiyama K et al: A girl with early-onset epileptic encephalopathy associated with microdeletion involving CDKL5. Brain Dev 2012; 34: 364-367.

20 Erez A, Patel AJ, Wang X et al: Alu-specific microhomology-mediated deletions in CDKL5 in females with early-onset seizure disorder. Neurogenetics 2009; 10: 363-369.

21 Sprovieri T, Conforti FL, Fiumara A et al: A novel mutation in the X-linked cyclindependent kinase-like 5 (CDKL5) gene associated with a severe Rett phenotype. Am J Med Genet A 2009; 149A: 722-725.

22 Artuso R, Mencarelli MA, Polli R et al: Early-onset seizure variant of Rett syndrome: definition of the clinical diagnostic criteria. Brain Dev 2010; 32: 17-24.

23 Bahi-Buisson N, Girard B, Gautier A et al: Epileptic encephalopathy in a girl with an interstitial deletion of Xp22 comprising promoter and exon 1 of the CDKL5 gene. Am J Med Genet B Neuropsychiatr Genet 2010; 153B: 202-207

24 Coppola G, Grosso S, Verrotti A, D'Aniello A, Pascotto A: Simultaneous onset of infantile spasms in monozygotic twins. Pediatr 2010; 43: 127-130.

25 Cordova-Fletes C, Rademacher N, Muller I et al: CDKL5 truncation due to a $\mathrm{t}(\mathrm{X} ; 2)(\mathrm{p} 22.1 ; \mathrm{p} 25.3)$ in a girl with X-linked infantile spasm syndrome. Clin Genet 2010; 77: 92-96.

26 Hadzsiev K, Polgar N, Bene J et al: Analysis of Hungarian patients with Rett syndrome phenotype for MECP2, CDKL5 and FOXG1 gene mutations. J Hum Genet 2011; 56: 183-187.

27 Masliah-Plachon J, Auvin S, Nectoux J, Fichou Y, Chelly J, Bienvenu T: Somatic mosaicism for a CDKL5 mutation as an epileptic encephalopathy in males. Am J Med Genet 2010; 152A: 2110-2111.

28 Mei D, Marini C, Novara F et al: Xp22.3 genomic deletions involving the CDKL5 gene in girls with early onset epileptic encephalopathy. Epilepsia 2010; 51: 647-654.

29 White R, Ho G, Schmidt S et al: Cyclin-dependent kinase-like 5 (CDKL5) mutation screening in Rett syndrome and related disorders. Twin Res Hum Genet 2010; 13 168-178.

30 Castrén M, Gaily E, Tengström C, Lähdetie J, Archer H, Ala-Mello S: Epilepsy caused by CDKL5 mutations. Eur J Paediatr Neurol 2011; 15: 65-69.

31 Melani F, Mei D, Pisano T et al: CDKL5 gene-related epileptic encephalopathy: electroclinical findings in the first year of life. Dev Med Child Neurol 2011; 53: 354-360. 
32 Sartori S, Polli R, Bettella E et al: Pathogenic Role of the X-Linked cyclin-dependent kinase-like 5 and aristaless-related homeobox genes in epileptic encephalopathy of unknown etiology with onset in the first year of life. J Child Neurol 2011; 26: 683-691.

33 Rademacher N, Hambrock M, Fischer $U$ et al: Identification of a novel CDKL5 exon and pathogenic mutations in patients with severe mental retardation, early-onset seizures and Rett-like features. Neurogenetics 2011; 12: 165-167.

34 Klein KM, Yendle S, Harvey A et al: A distinctive seizure type in patients with CDKL5 mutations: hypermotor-tonic-spasms sequence. Neurology 2011; 76: 1436-1438.

35 Bartnik M, Derwinska K, Gos M et al: Early-onset seizures due to mosaic exonic deletions of CDKL5 in a male and two females. Genet Med 2011; 13: 447-452.

36 Stalpers XL, Spruijt L, Yntema HG, Verrips A: Clinical phenotype of 5 females with a CDKL5 mutation. J Child Neurol 2012; 27: 90-93.

37 Liang JS, Shimojima K, Takayama R et al: CDKL5 alterations lead to early epileptic encephalopathy in both genders. Epilepsia 2011; 52: 1835-1842.

38 Intusoma U, Hayeeduereh F, Plong-On $\mathrm{O}$ et al: Mutation screening of the CDKL5 gene in cryptogenic infantile intractable epilepsy and review of clinical sensitivity. Eur J Paediatr Neurol 2011; 15: 432-438.

39 Hanefield F: The clinical pattern of the Rett syndrome. Brain Dev 1985; 7: 320-325.

40 Neul JL, Kaufmann WE, Glaze DG et al: Rett syndrome: revised diagnostic criteria and nomenclature. Ann Neurol 2010; 68: 944-950.
41 Bebbington A, Anderson A, Ravine D et al: Investigating genotype-phenotype relationships in Rett syndrome using an international dataset. Neurology 2008; 70: 868-875.

42 WHO multicentre growth reference study group. WHO Motor Development Study: windows of achievement for six gross motor development milestones. Acta Paediatr 2006; 95: 86-95.

43 Kankirawatana $\mathrm{P}$, Leonard $\mathrm{H}$, Ellaway $\mathrm{C}$ et al: Early progressive encephalopathy in boys and MECP2 mutations. Neurology 2006; 67: 164-166.

44 Kortüm F, Das S, Flindt M et al: The core FOXG1 syndrome phenotype consists of postnatal microcephaly, severe mental retardation, absent language, dyskinesia, and corpus callosum hypogenesis. J Med Genet 2011; 48: 396.

45 Marangi G, Ricciardi S, Orteschi D et al: The Pitt Hopkins syndrome: report of 16 new patients and clinical diagnostic criteria. Am J Med Genet 2011; 155A: 1536-1545.

46 Scheffer I, Brett E, Wilson J, Baraitser M: Angelman's syndrome. J Med Genet 1990; 27: 275.

47 Allanson JE, Hennekam R, Moog U, Smeets EE: Rett syndrome: a study of the face. Am J Med Genet 2011; 155A: 1563-1567.

48 Fehr S, Bebbington A, Ellaway C, Rowe P, Leonard H, Downs J: Altered attainment of developmental milestones influences the age of diagnosis of Rett syndrome. J Child Neurol 2011; 26: 980-987.

49 Leonard H, Bower C: Is the girl with Rett syndrome normal at birth? Dev Med Child Neurol 1998; 40: 115-121.

Supplementary Information accompanies the paper on European Journal of Human Genetics website (http://www.nature.com/ejhg) 